\title{
Teeth, tools and human becoming
}

\section{Karenleigh A. Overmann}

Keble College, Parks Road, University of Oxford, Oxford, England, OX1 3PG, UK

e-mail: karenleigh.overmann@keble.ox.ac.uk

As Bruner \& Lozano (JASs forum 2014, vol. 92: 273) noted, Neandertal teeth are distinguished from those of Homo sapiens by, among several characteristics, the size and "shovel-shaped' morphology" of their incisors (Bailey, 2006, p. 257), as well as a distinctive wear pattern indicating their use as tools or a 'third hand', often to the point of "obliterat[ing]" them while leaving the molars only "lightly worn" (Bailey \& Liu, 2010, p. 20). Anterior tooth size and shoveling, traits Neandertals shared with ancestral Homo, were reduced in early $H$. sapiens, who showed a concomitant reduction in wear associated with third-hand use (Bailey, 2006; Le Cabec et al., 2013). Bruner \& Lozano (2014) used the latter characteristic, wear-patterns differences, to argue that Neandertals "may have suffered a mismatch between cultural and neural complexity, in which additional anatomical elements were involved as a body interface to overcome insufficient visuospatial integration processes" (p. 276). Visuospatial integration, the synthesis of visual and spatial sensory inputs, facilitates motor movements in space, ranging from small to large muscles (e.g., handeye coordination and running) and near to far distances (peripersonal and extrapersonal space). In extinct species, since we cannot watch them move, visuospatial integration can only be assessed indirectly, through the behavioral traces left on material culture and fossils, and morphological change in both over time.

Bruner \& Lozano (2014) offer an interdisciplinary synthesis of three disparate domains: data on archaic teeth, the functional implications of gross neuroanatomy as determined from fossils and endocasts, and theories of embodiment and extended mind. Archaic teeth data support the hypothesis that Neandertals used their anterior teeth as a third hand to a greater extent than did H. sapiens (Bailey, 2006; Bailey \& Liu, 2010; Le Cabec et al., 2013), and such behavioral differences, given sufficient time, have the potential to cause morphological change, a factor in reproductive isolation and speciation (e.g., Overmann $\&$ Coolidge, 2013). In the case under discussion, behavioral differences are consistent with morphological change: $H$. sapiens lost several primitive characteristics, particularly shoveling and size in the anterior teeth, that Neandertals retained (Bailey, 2006; Le Cabec et al., 2013). Evolutionary dental reduction has long been associated with technological change thought to have shifted food processing from mouth to hands to tools (e.g., Holloway, 1967; Molnar, 1972): the "progression reduction in [tooth] size [seen] through the Pleistocene ... perhaps implies that the larger teeth were not as necessary for survival or even for day to day use as they had been in earlier times" (Dahlberg, 1963, p. 240). Further, shovel-shaped teeth have increased "strength and mass ... and are decidedly advantageous to the possessor," associated with reduced breakage of the upper incisors in modern populations with more shoveling than the average today but still less than the Neandertals had (Dahlberg, 1963, p. 244). Larger, stronger teeth would have made third-hand behavior less risky for Neandertals, and third-hand behavior likely explains why Neandertals retained the primitive dental features.

The decrease in $H$. sapiens third-hand use of the anterior teeth and attendant dental reduction were presumably offset by an increased use of technology (a circumstance that might not be 
discernable in the archaeological record because the period in question lies beyond the preservation horizon for most perishable materials). Answering the question of whether weaker teeth necessitated increased tool use or increased tool use led to weaker teeth is not critical: there is no way of determining which of the two conditions occurred first; they would have been mutually reinforcing in any case, and it is the trajectory of change that is important. The trajectory intensified $H$. sapiens' need to incorporate tools into its behavioral repertoire, tools that functioned as integral parts of the body through neural responses to tools: for example, neurons controlling finger movements react to a grasped tool as if it were part of the hand (Vaesen, 2012). When a tool extends the arm's reach, the body-schema 'map' of the body's physical extent and disposition changes to include the tool, and visual space is remapped so that objects within the extended reach of the tool seem nearer (Bonifazi et al., 2007; Farnè \& Làdavas, 2000; Maravita et al., 2001, 2002; Maravita \& Iriki, 2004).

The neural basis for tool use (Orban \& Caruana, 2014), as well as motor control and sensory integration, are associated with the parietal lobe, whose morphological change constitutes a significant difference in the gross neuroanatomy of the two human species (Bruner, 2004, 2010; Bruner et al., 2003, 2014; Friess, 2010), highlighted by Coolidge (2014) in his commentary on Bruner \& Lozano's (2014) proposal. Parietal expansion changed the overall shape of the $H$. sapiens brain to a more globular form and informed subsequent neural reorganizations that would have facilitated interconnectivity and interaction, yielded faster signaling rates, and avoided evolutionary trade-offs associated with larger brains (e.g., Azevedo et al., 2009; Gibson, 1991; Kaas, 2000; Rilling \& Insel, 1999; Seghier, 2012). Because of the relation between brain region size and adaptive use of its functions, the increase in lobe volume suggests that parietal functions were important to H. sapiens' ability to exploit its niche.

Two parietal areas highlighted by Bruner \& Lozano (2014) were the intraparietal sulcus
(IPS) and precuneus. The IPS, a portion of the parietal lobe located laterally on the cortical surface, has been implicated in a variety of cognitive functions, including motor planning, perceptual-motor coordination, various forms of attention, working memory, numerosity and calculation, spatial representation, spatial coordinate transformation, mental rotation, aspects of long-term memory and language, and intent interpretation (Ansari et al., 2005; Cantlon et al., 2006; Diester \& Nieder, 2008; Hamilton \& Grafton, 2006; Hubbard et al., 2009; Koenigs et al., 2009; Orban et al., 2006). The precuneus, a superior medial portion of the parietal lobe, has been implicated in attention, visuospatial processing, self-consciousness, self-representation, and various forms of memory (Addis et al., 2007; Cavanna \& Trimble, 2006; Lou et al., 2004; Zhang \& Li, 2012).

Bruner \& Lozano (2014) found the IPS and precuneus "particularly interesting" because of their role in hand-eye coordination and visuospatial integration (p. 274), speculating that Neandertals had a "mismatch" or insufficiency in its functioning (p. 276). However, in Neandertals visuospatial integration would have been, as it would have been in contemporary and is today in extant $H$. sapiens, a product of the interaction between the functionality of their brains, the physical characteristics and behaviors of their bodies, and the materiality they manipulated in specific environmental and social contexts. That is, taking cognition as a complex system composed of interacting elements - brain, body and materiality (Malafouris, 2013) - Neandertal cognition was well suited to its adaptive context. Whether or not Neandertal visuospatial integration differed from that of $H$. sapiens, it was certainly sufficient for them to demonstrate impressive competency and skill, as attested by "the complexity of the Levallois technique, the use of compound technology, and the very fact of their long-term survival across wide geographic and climatic ranges" (Overmann \& Coolidge, 2013, p. 94).

Further, Neandertal mastery of the demanding Levallois technique seems compelling evidence against their having been particularly 
deficient in hand-eye coordination, though Bruner \& Lozano (2014) are likely correct in asserting that the critical differences between the two human species lie in their respective parietal functionality. Together, increased tool use, parietal functionality for tool manipulation (particularly the incorporation of tools into the bodyschema), and the involvement of the precuneus in the sense of self suggest that $H$. sapiens may have had a different relationship with material culture than Neandertals did, incorporating it more completely into their cognitive system. For $H$. sapiens, material objects became part of the body and hence the mind. Integrated neurally and in contact with hands and other anatomy, material objects extended body and mind physically and distributed them spatially and temporally, as theories of embodiment and extended mind suggest. For example, Malafouris (2008) has proposed that material objects functioning as social markers (e.g., personal ornaments) might extend a concept of self through time: ornaments "transform the phenomenological self-as-subject to a social self-as-object" (Malafouris, 2008, p. 408), anchoring the self with the meaning they instantiate and extending the self into the past and future through their durability and continuity (Malafouris, 2013). Because of differences in parietal functionality, $H$. sapiens brains and bodies may have integrated materiality into their cognitive system in a deeper, more profound manner. Visuospatial integration, then, seems something that would improve with increased tool use, a practice effect reinforcing the propensity to use tools, yielding over time both cultural and biological change.

From a developmental perspective, proprioceptive, tactile, and visual awareness of the hand is integrated with cognitive processes like object recognition and spatiality in early childhood, intermodal integration that is sensitive to tool use and changes in body posture (Bremmer $\&$ Cowie, 2013). This is not to suggest an ontogenetic recapitulation but rather to note that hominid phylogenetic history includes significant postural change: bipedalism, for example, freed the hands for tool use in the species ancestral to both $H$. sapiens and Neandertals, "lighting a fuse on a long process that entrained many other parts of the human body and many other faculties as it unfolded" (Tallis, 2003, p. 6). In addition to the renegotiation of sensorimotor faculties that bipedalism implies, the increased ability to engage materiality through the hand would have opened up cascading possibilities for further change. The descendant species then perhaps differed not in kind but in the degree to which the use of tools yielded subsequent change to behavior, bodies, and brains. Thus, the critical difference between the two species may not have been hand-eye coordination per se, since Neandertals demonstrated considerable skill in this regard, but a more pervasive prosthetic adaptation by $H$. sapiens.

The differences between the two species suggests, in turn, that understanding human becoming requires, as Bruner \& Lozano (2014) have set out to do, a holistic consideration of brains (paleoneurology), bodies (paleontology), and materiality (archaeology) as an interacting system, examining what indicators of change might reveal about the functionality of the constituent elements in interaction with one another and the development of complexity in systemic behaviors.

\section{References}

Addis D.R., Wong A.T. \& Schacter D.L. 2007. Remembering the past and imagining the future: common and distinct neural substrates during event construction and elaboration. Neuropsychologia, 45: 1363-1377.

Ansari D., Garcia N., Lucas E., Hamon K. \& Dhital B. 2005. Neural correlates of symbolic number processing in children and adults. Neuroreport, 16: 1769-1773.

Azevedo F.A.C., Carvalho L.R.B., Grinberg L.T., Farfel J.M., Ferretti R.E.L., Leite R.E.P., Filho W.J., Lent R. \& Herculano-Houzel S. 2009. Equal numbers of neuronal and nonneuronal cells make the human brain an isometrically scaled-up primate brain. J. Comp. Neurol., 513: 532-541. 
Bailey S. E. \& Liu W. 2010. A comparative dental metrical and morphological analysis of a Middle Pleistocene hominin maxilla from Chaoxian (Chaohu), China. Quat. Int., 211: 14-23.

Bailey S.E. 2006. Beyond shovel-shaped incisors: Neandertal dental morphology in a comparative context. Period. Biol., 108: 253-267.

Bonifazi S., Farnè A., Rinaldesi M.L. \& Làdavas E. 2007. Dynamic size-change of peri-hand space through tool-use: spatial extension or shift of the multi-sensory area. J. Neuropsychol., 1: 101-114.

Bremmer A.J. \& Cowie D. 2013. Developmental origins of the hand in the mind, and the role of the hand in the development of the mind. In Z. Radman (ed): The Hand, an Organ of the Mind: What the Manual Tells the Mental, pp. 27-55. MIT Press, Cambridge, MA.

Bruner E. 2004. Geometric morphometrics and paleoneurology: brain shape evolution in the genus Homo. J. Hum. Evol., 47: 279-303.

Bruner E. 2010. Morphological differences in the parietal lobes within the human genus. Curr. Anthropol., 51: S77-S88.

Bruner E., Manzi G. \& Arsuaga J.L. 2003. Encephalization and allometric trajectories in the genus Homo: evidence from the Neandertal and modern lineages. Proc. Natl. Acad. Sci. U.S.A., 100: 15335-15340.

Bruner E., Rangel de Lázaro G., de la Cuétara J.M., Martín-Loeches M., Colom R. \& Jacobs H.I.L. 2014. Midsagittal brain variation and MRI shape analysis of the precuneus in adult individuals. J. Anat., 224: 367-376.

Cantlon J.F., Brannon E.M., Carter E.J. \& Pelphrey K.A. 2006. Functional imaging of numerical processing in adults and 4-y-old children. PLoS Biol., 4: 844-854.

Cavanna A.E. \& Trimble M.R. 2006. The precuneus: a review of its functional anatomy and behavioural correlates. Brain, 129: 564-583.

Coolidge F.L. 2014. The exaptation of the parietal lobes in Homo sapiens. J. Anthropol. Sci., 92: 295-298.

Dahlberg A.A. 1963. Dental evolution and culture. Hum. Biol., 35: 237-249.
Diester I. \& Nieder A. 2008. Complementary contributions of prefrontal neuron classes in abstract numerical categorization. J. Neurosci., 28: 7737-7747.

Farnè A. \& Làdavas E. 2000. Dynamic sizechange of hand peripersonal space following tool use. Neuroreport, 11: 1645-1649.

Friess M. 2010. Parietal expansion during later hominin evolution, and the validity of $H$. heidelbergensis-A quantitative approach. Bulletin Der Schweizerischen Gesellschaft Für Anthropologie, 16: 55-61.

Gibson K.R. 1991. Myelination and behavioral development: a comparative perspective on questions of neoteny, altriciality and intelligence. In K.R. Gibson \& A.C. Petersen (eds): Brain Maturation and Cognitive Development: Comparative and Cross-Cultural Perspectives, pp. 29-63. Aldine Transaction, Piscataway.

Hamilton A.F. de C. \& Grafton S.T. 2006. Goal representation in human anterior intraparietal sulcus. J. Neurosci., 26: 1133-1137.

Holloway R.L. 1967. Tools and teeth: some speculations regarding canine reduction. Am. Anthropol., 69: 63-67.

Hubbard E.M., Piazza M., Pinel P. \& Dehaene S. 2009. Numerical and spatial intuitions: a role for posterior parietal cortex? In L. Tommasi, M.A. Peterson \& L. Nadel (eds): Cognitive Biology: Evolutionary and Developmental Perspectives on Mind, Brain and Behavior, pp. 221-246. MIT Press, Cambridge, MA.

Kaas, J.H. 2000. Why is brain size so important: design problems and solutions as neocortex gets bigger or smaller. Brain and Mind, 1: 7-23.

Koenigs M., Barbey A.K., Postle B.R. \& Grafman J. 2009. Superior parietal cortex is critical for the manipulation of information in working memory. J. Neurosci., 29: 14980-14986.

Le Cabec A., Gunz P., Kupczik K., Braga J. \& Hublin J.-J. 2013. Anterior tooth root morphology and size in Neandertals: taxonomic and functional implications. J. Hum. Evol., 64: 169-193.

Lou H.C., Luber B., Crupain M., Keenan J.P., Nowak M., Kjaer T.W., Sackeim H.A. \& Lisanby S.H. 2004. Parietal cortex and 
representation of the mental self. Proc. Natl. Acad. Sci. U.S.A., 101: 6827-6832.

Malafouris L. 2008. Beads for a plastic mind: the 'blind man's stick' (BMS) hypothesis and the active nature of material culture. Camb. Archaeol. J., 18: 401-414.

Malafouris L. 2013. How Things Shape the Mind: A Theory of Material Engagement. MIT Press, Cambridge, MA.

Maravita A. \& Iriki A. 2004. Tools for the body (schema). Trends Cogn. Sci., 8: 79-86.

Maravita A., Husain M., Clarke K. \& Driver J. 2001. Reaching with a tool extends visual-tactile interactions into far space: evidence from crossmodal extinction. Neuropsychologia, 39: 580-585.

Maravita A., Spence C., Kennett S. \& Driver J. 2002. Tool-use changes multimodal spatial interactions between vision and touch in normal humans. Cognition, 83: B25-B34.

Molnar S. 1972. Tooth wear and culture: a survey of tooth functions among some prehistoric populations. Curr. Anthropol., 13: 511-526.

Orban G.A. \& Caruana F. 2014. The neural basis of human tool use. Frontiers in Psychology, 5: 1-12.
Orban G.A., Claeys K., Nelissen K., Smans R., Sunaert S., Todd J.T. Wardak C., Durand J.B. \& Vanduffel W. 2006. Mapping the parietal cortex of human and non-human primates. Neuropsychologia, 44: 2647-2667.

Overmann K.A. \& Coolidge F.L. 2013. Human species and mating systems: Neandertal-Homo sapiens reproductive isolation and the archaeological and fossil records. J. Anthropol. Sci., 91: 91-110.

Rilling J.K. \& Insel T.R. 1999. Differential expansion of neural projection systems in primate brain evolution. Neuroreport, 10: 1453-1459.

Seghier M.L. 2012. The angular gyrus: multiple functions and multiple subdivisions. Neuroscientist, 20: 1-19.

Tallis R. 2003. The Hand: A Philosophical Inquiry into Human Being. Edinburgh University Press, Edinburgh.

Vaesen K. 2012. The cognitive bases of human tool use. Behav. Brain Sci., 35: 203-218.

Zhang S. \& Li C.S.R. 2012. Functional connectivity mapping of the human precuneus by resting State fMRI. NeuroImage, 59: 3548-3562. 